\title{
Carcinomas in inflammatory bowel disease: a narrative review on diagnostic imaging techniques
}

\author{
Federica Furfaro ${ }^{1}$, Arianna Dal Buono ${ }^{1}$, Claudio Sicuso ${ }^{2}$, Mariangela Allocca ${ }^{3}$, Ferdinando D'Amico,4, \\ Alessandra Zilli ${ }^{3}$, Gionata Fiorino ${ }^{1,4}$, Roberto Gabbiadini ${ }^{1}$, Silvio Danese ${ }^{3}$ \\ ${ }^{1}$ IBD Center, Humanitas Research Hospital-IRCCS, Rozzano, Milan, Italy; ${ }^{2}$ Department of Radiology, Humanitas Research Hospital-IRCCS, \\ Rozzano, Milan, Italy; ${ }^{3}$ Gastroenterology and Endoscopy, IRCCS Ospedale San Raffaele and Vita-Salute San Raffaele University, Milan, Italy; \\ ${ }^{4}$ Department of Biomedical Sciences, Humanitas University, Pieve Emanuele, Milan, Italy \\ Contributions: (I) Conception and design: F Furfaro, A Dal Buono; (II) Administrative support: None; (III) Provision of study materials or patients: \\ None; (IV) Collection and assembly of data: None; (V) Data analysis and interpretation: None; (VI) Manuscript writing: All authors; (VII) Final \\ approval of manuscript: All authors. \\ Correspondence to: Federica Furfaro, MD, PhD. IBD Center, Humanitas Research Hospital-IRCCS, Via Manzoni 56, 20089 Rozzano, Milan, Italy. \\ Email: Federica.furfaro@humanitas.it.
}

Objective: We aimed, therefore, to review the current evidence on imaging modalities and carcinomas overlapping IBD.

Background: Patients affected by inflammatory bowel diseases (IBD) are at increased risk for developing both gastrointestinal and extra-intestinal cancers. The subtype of IBD, namely Crohn's disease or ulcerative colitis, the location, the activity, the extent, and the duration of the disease determine this risk. Standardized surveillance programs based on imaging techniques exist only for colorectal cancer, where colonoscopy is the milestone of early detection. Clarification is needed on whether different imaging modalities might be adopted in the algorithms for screening and diagnosis of cancers in IBD patients.

Methods: PubMed was searched up to July 2021 to identify relevant studies investigating the accuracy of imaging techniques in identifying carcinomas in IBD patients. The following text words and corresponding Medical Subject Heading/Entree terms were used: "imaging”, "computed tomography", "magnetic resonance imaging", "inflammatory bowel disease", "adenocarcinoma" and "cancer".

Conclusions: Currently dye-chromoendoscopy (DCE) is established as the gold standard diagnostic modality for the detection of dysplasia in IBD, with a demonstrated superiority compared to white-light endoscopy. Two main radiological patterns have been described at cross-sectional imaging for both colorectal cancer and small bowel adenocarcinoma. The first subtype is characterized by a tissue mass, while the second subtype recognizes a circumferential thickening with or without the stricturing of the lumen. The diagnostic sensitivity, specificity, and accuracy of cross-sectional imaging techniques for the detection of carcinomas in the context of IBD are largely unknown and scarcely investigated. The definition of surveillance programs based on different imaging methods is warranted.

Keywords: Imaging; bowel ultrasound (BUS); inflammatory bowel disease (IBD); carcinomas; surveillance

Submitted Jul 24, 2021. Accepted for publication Dec 07, 2021.

doi: $10.21037 /$ cco-21-98

View this article at: https://dx.doi.org/10.21037/cco-21-98 


\section{Introduction}

Inflammatory bowel diseases (IBD) are chronic, relapsing-remitting disorders of the gastrointestinal tract characterized by inflammation within the bowel wall $(1,2)$. IBD patients are at increased risk for several gastrointestinal and extra-intestinal cancers depending on the subtype of IBD [i.e., Crohn's disease (CD), ulcerative colitis, or unclassified IBD], the location, the activity, the extent, and duration of the disease (3-5). Beyond inflammation as a trigger for carcinogenesis, some differences in the risk of cancer between patients with IBD and the general population may be explained by the prolonged use of immunosuppressants, especially steroids, and by personal or lifestyle factors such as age, smoking, concomitant primary sclerosing cholangitis (PSC), or family history for cancer in first-degree relatives (6). The most frequent location of gastrointestinal cancer in patients with longstanding IBD is the large bowel (i.e., colon, rectum), whereas among the other cancers associated with IBD, small bowel adenocarcinomas, anal cancer, and carcinoid tumors occur less commonly (3-5). In the last decade, improved screening strategies, mainly through surveillance colonoscopies, as recommended by the SCENIC consensus (7) have led to elevated detection rates of dysplasia and decreasing rates of colon-rectal cancer (CRC) (8). On the other hand, crosssectional imaging has emerged as an additional screening modality for malignancies in case of unusual symptoms and suspected complications in IBD patients $(9,10)$. Despite colonoscopy with biopsy is currently regarded as the gold standard for CRC surveillance in IBD, some districts where cancer might eventually develop, above all the small bowel, are difficult to be explored by endoscopists (7). Besides, imaging techniques hold the advantage to spare frequent invasive procedures and to inform about local and distant metastatic disease and about the therapeutic response. Gastroenterologists and radiologists are, therefore, strictly connected in the management of IBD-related cancers both in the diagnosis and follow-up. Considering the lack of guidelines instructing on the adoption of cross-sectional imaging in the field of surveillance and prevention of carcinomas in IBD patients, dedicated physician often indicate the technique based on their clinical experience and a comprehensive summary of the current evidence in this field is strongly needed. In this review we aim, therefore, to examine and summarize the current evidence and the latest advances in the field of imaging techniques including mainly computed tomography (CT), magnetic resonance imaging (MRI), and bowel ultrasound (BUS) in the diagnosis of gastrointestinal cancers occurring in IBD patients. We present the following article in accordance with the Narrative Review reporting checklist (available at https://cco.amegroups.com/article/view/10.21037/cco-2198/rc).

\section{Methods}

PubMed was searched up to July 2021 to identify relevant studies investigating the accuracy of imaging techniques in identifying carcinomas in IBD patients. The following text words and corresponding Medical Subject Heading/Entree terms were used: "imaging", "computed tomography", "magnetic resonance imaging", "inflammatory bowel disease", "adenocarcinoma" and "cancer".

\section{Colorectal cancer}

IBD patients have a risk of developing colorectal cancer that ranges from 1.5 to 2.4 times greater than in the general population $(11,12)$. The risk of IBD-associated CRC becomes consistent after 8 years from symptoms appearance and increases linearly over the nest years, being higher in patients with extensive colitis (7). Compared to sporadic CRC, IBD-associated CRC has several distinctive features: it affects younger patients, it is more frequently located in proximal segments, the histological sub-type with signetring cells can be more often encountered $(13,14)$.

\section{Endoscopic technologies}

The standard endoscopic management of colorectal lesions involves firstly the detection of the lesion itself and, thereafter, the characterization based on the mucosal surface and vessels' architecture. As reported in a recent meta-analysis including five observational studies with a total of more than 7,000 IBD patients, the endoscopic surveillance in IBD is associated with a reduction in CRC occurrence, morbidity and death, as well as an increased recognition of early-stage CRC (15). Currently dyechromoendoscopy (DCE) is established and endorsed by meta-analysis as the gold standard diagnostic modality for the detection of dysplasia in IBD, with a demonstrated superiority compared to white-light endoscopy (WLE) (7). Indeed, numerous recent randomized clinical trials have proven that DCE enables a 2 -fold higher detection of dysplasia than conventional white-light colonoscopy $(16,17)$. 

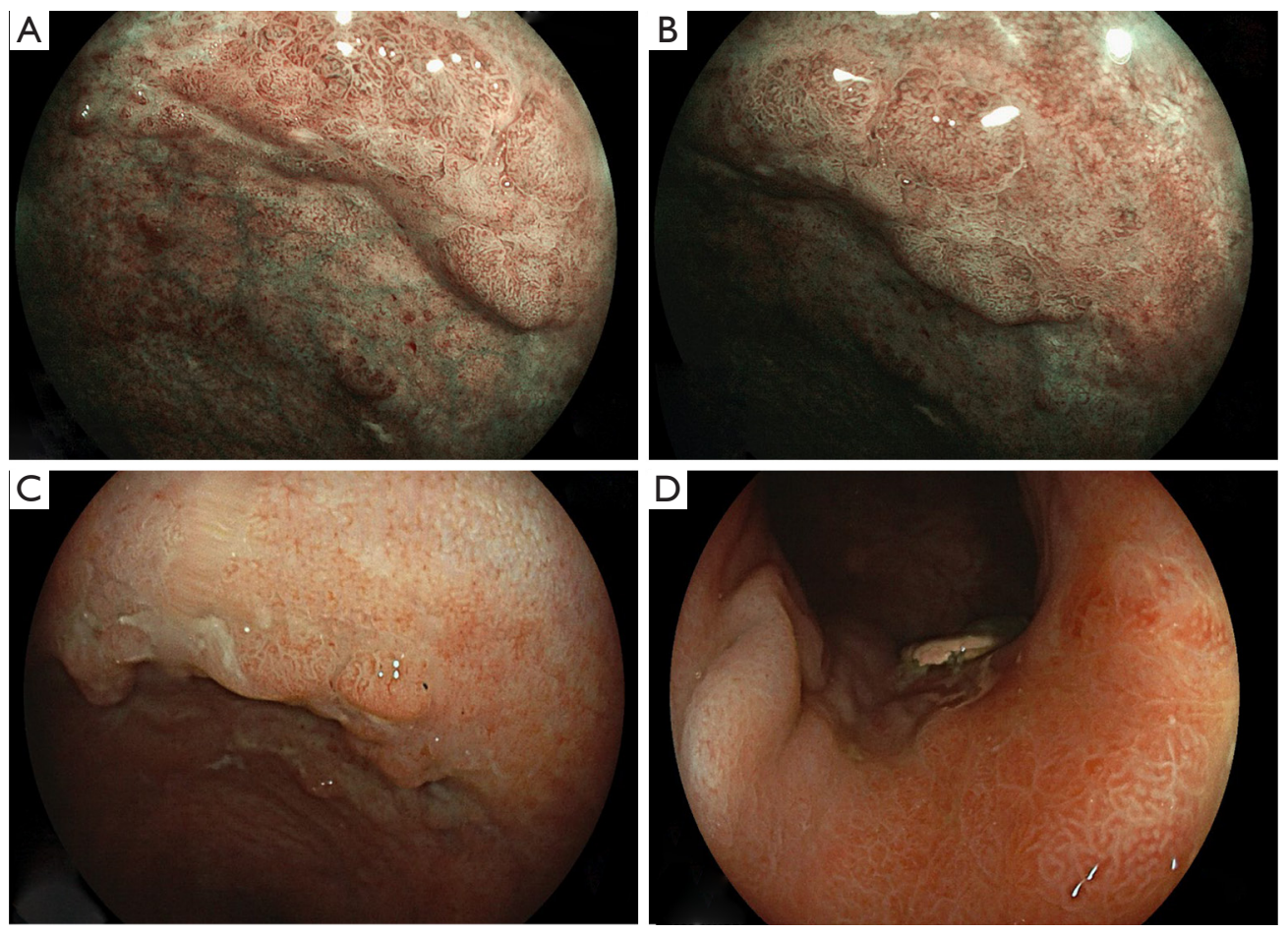

Figure 1 An example of virtual chromoendoscopy and high definition wight light endoscopy of a neoplastic lesion of the rectum. (A,B) Virtual chromoendoscopy with BLI of a rectal lesion, pit pattern IV according to Kudo classification with elongated and branched pits and some central area of loss of the normal glandular pattern; (C,D) the same lesion at wight-light endoscopy. The histology after surgical resection revealed an adenocarcinoma $\mathrm{p} T 1$.

As concerns technical aspects, chromoendoscopy applies dyes to the colonic mucosa creating a contrast enhancement of the mucosal patterns, that increases the visualization of suspicious areas or lesions $(18,19)$. Traditionally, methylene blue and indigo carmine represent the most commonly agents employed: the application is via a catheter or water jet channel $(18,19)$. Newly, emerging data have shown that virtual chromoendoscopy (VCE) is as effective as chromoendoscopy in revealing dysplastic lesions in IBD patients $(20,21)$. In details, according to a recent metaanalysis that included more than 3,000 studies accounting for a total of 1,328 patients revealed no statistically significant difference between VCE and DCE in the accuracy of neoplastic surveillance (risk ratio $0.77 ; 95 \%$ CI: 0.55-1.08) (21). As concerns the typical endoscopic characteristics of CRC in IBD patients, they are classified according to the Paris classification for their morphology as polypoid or non-polypoid, and according to the Kudo classification for the glandular pit pattern $(22,23)$. In details, type I pits appear as roundish, type II pits appear as stellar, type III-s pits are small and tubular and type III-L are also tubular but larger, finally type IV identifies branchlike and/or gyrus-like pits and type $\mathrm{V}$ identifies a fully destructured pattern. The classes III-V detects neoplastic and malignant changes of the lesion. Figure 1 shows an example of a neoplastic lesion at WLE and VCE of a patient affected by UC.

\section{Cross-sectional imaging in CRC}

Given that endoscopy represents the backbone of the diagnosis of CRC in IBD, cross-sectional imaging techniques remain unbeaten with respect to staging of both local and distant disease (24). Additionally, MRI has a prominent role in the local staging of rectal cancer (25). With respect to CT imaging, there are distinctive patterns of wall thickening that allow to differentiate between a highly suspicious malignancy, that will appear as an irregular and heterogeneous focal thickening, whereas a symmetrical and homogeneous diffuse thickening will rather suggest the inflammatory location of $\operatorname{IBD}(26,27)$. However, this distinction is not always obvious, since 
in many cases of IBD, chronic and acute inflammatory processes coexist with some asymmetrical aspects that can confound the radiologist $(26,27)$. Studies investigating the CT features of IBD-related CRC are scanty on mostly in small cohorts. CT scan is essential in the diagnostic process of CRC arising in or above strictures of the colon, that cannot be reached and accurately evaluated by the endoscopist (28). Additionally, if the IBD-related CRC progresses from a flat colonic lesion it may be undetected by WLE and evident only at CT scan in up to $50 \%$ of the cases (28). Some authors have described two main radiological patterns: a first one of a typical tissue mass with an intra- or/and extra-luminal growth, and a second radiological pattern characterized by a circumferential thickening of the wall eventually stricturing the lumen (28). The thickened colorectal wall can present irregular rims and ulcerations when concomitant active IBD is present (28). Notably, in a retrospective cohort of 17 IBD patients, a significant association was found between the presence of signet ring cells at histology and the type 2 tumor pattern at CT $(\mathrm{P}=0.009)$, and the presence of free-fluid effusion was assessed as independent variable for the presence of signet ring cells $(\mathrm{P}=0.01)$ (28). General features suggesting malignancy at $\mathrm{CT}$ scan are an asymmetric mural thickening, a focal loss of wall stratification, and mural thickening greater than $1.5 \mathrm{~cm}$ (29). Newly, the CT texture analysis has been proposed as additional tool in the differential diagnosis of tumorous and non-tumorous colonic lesions with an accuracy of $94.3 \%$ for CRC vs. IBD locations compared to conventional three-phase CT imaging (27). Finally, the use of PET-CT has been described in a single case of an IBD patient affected by rectal cancer where this technique overestimated the tumor extension (30). The increased uptake by the sites of active IBD lead to a falsely positive interpretation of the imaging (30). Regarding the application of MRI for colonic CRC in IBD, it has been demonstrated that steadily high signal intensity at diffusion weighted images (DWI), specifically with b-values over $3,000 \mathrm{~s} / \mathrm{mm}^{2}$, can indicate neoplastic lesions. This cut-off has been suggested to discriminate neoplasia from mere inflammation in the ileo-cecal region (31). In a further retrospective study, carcinomas showed significantly lower mean apparent diffusion coefficient values than IBD $(\mathrm{P}<0.001)(32)$. MRI is currently recommended as radiological assessment for the staging of rectal cancer (25), with a sensitivity of $95-100 \%$ for tumor detection and accuracy ranging from $76 \%$ to $93 \%$ for the tumor infiltration of the rectal wall and mesorectal tissue in case of sporadic rectal cancer (25). Rare studies have been performed in IBD-related rectal cancer. In a retrospective series of thirteen IBD patients the same above-mentioned sub-classification of radiological patterns (type 1 and type 2) has been described for rectal IBD-related cancer (10). Interestingly, $53.8 \%$ of the cases occurred in absence of abscess or fistulas (10). The authors suggested the adoption of T2-weighted and diffusion-weighted (DW-) MRI to improve the detection of rectal cancer in IBD patients, reaching $92 \%$ the forms that showed high signal intensity on T2-weighted and $100 \%$ the forms that showed high signal on DW-MRI with restricted diffusion on apparent diffusion coefficient plot (10).

\section{BUS in CRC}

Trans-abdominal BUS is being increasingly recognized as an accurate tool for the diagnosis and monitoring of IBD and its use is growing among dedicated gastroenterologist and radiologist due to its non-invasiveness, low costs and reproducibility (33). A location of active IBD and CRC may have similar ultrasonographic findings such as bowel wall thickening $>3 \mathrm{~mm}$, also with irregular rims, and locoregional lymphadenopathies (33). Emerging data about the BUS appearance of CRC reported that the loss of ultrasonographic stratification can be present in up to $97.4 \%$ of the cases and with a statistically significant difference compared to active IBD findings $(\mathrm{P}<0.001)(33)$. Whereas no statistically significant difference was found between IBD and CRC patients as concerns Color Doppler features (34). Besides, the authors found that a disordered and a heterogeneous contrast-enhancement was as specific as $92.3-100 \%$ in predicting CRC at BUS (34). Based on these data the authors suggested contrastenhanced ultrasound (CEUS) as an accurate alternative diagnostic method to CT and MRI, being able to display the perfusion difference between inflammatory processes and tumors, without the drawbacks of high costs, radiation exposure, and nephrotoxicity (34). No further studies have specifically investigated BUS for CRC detection, diagnosis or management yet.

\section{Small bowel adenocarcinoma}

Small-bowel adenocarcinoma (SBA) is considered as a rare tumor in the general population. In case of IBD, only CD represents a risk factor for SBA, with 20 to 30 times increased odds of developing compared to non-affected 
subjects, as reported in population-based meta-analyses (35). A longer course of disease, more than 8 years, is also associated with an increased risk, with an estimated incidence that can reach 0.5 per 1,000 patient-years $(35,36)$. The carcinogenesis follows the sequence from dysplasia to adenocarcinoma: in those patients where dysplasia is encountered in ileal biopsies this process is thought to have started $(3,35)$. Both cross-sectional imaging video capsule or double balloon enteroscopy (DBE) are available for the diagnosis of SBA.

\section{Video capsule and balloon enteroscopy}

Since their recent development, DBE and video capsule endoscopy (VCE) allow the direct visualization of small bowel mucosa in its whole length (37). The main strength of DBE over VCE represents the possibility of biopsy and eventually any required therapeutic intervention. Therefore, in case of lesions suspicious for cancer or in any case in which a tissue pathological examination is required, DBE is indicated (37). Concerning technical aspects, the procedure requires a polyethylene glycol-based bowel preparation and fasting. The endoscopic aspect of SBA is usually of an ulcerated tumor within scarred or inflamed tissue (38). As concerns VCE, the retaining of the pill endoscopy camera has been described in literature in a case of CD complicated by an obtruding SBA, with its remotion through enterotomy at exploratory laparotomy (39). Therefore, this procedural risk has to be taken into account. In details, the incidence of capsule retention varies upon the initial indication, being estimated around $1.5 \%$ when performed for suspected $\mathrm{CD}$ and from $5 \%$ to $13 \%$ when performed for CD surveillance (40). Considering the risk of capsule retention, in patients with proven $\mathrm{CD}$, cross-sectional imaging or patency capsule, that is to say is a dissoluble device that allows to verify the adequate patency of the intestine, is suggested before proceeding with capsule endoscopy (41). The diagnostic work-up of suspicious strictures of the small bowel remains challenging, especially since the clinical presentation and imaging findings resemble an inflammatory flare of CD. Currently, there is no consensus on small bowel surveillance in $\mathrm{CD}$ and the mortality from SBA remains consistently high with an expected 5-year overall survival of $35 \%$ (42). A personalized approach can be attempted in patients at higher risk for SBA, but extensive and stricturing lesions make enteroscopy technically difficult. Indeed, procedural interruption due to inaccessible stricture has been reported in $46.5 \%$ of the cases in a recent multi-center, prospective study of CD patients at high-risk of SBA, defined as long-term small bowel disease (43). In this cohort, the authors assessed a sensitivity rate for SBA endoscopic screening by $33 \%$ (43).

\section{Cross-sectional imaging}

Radiological findings on CT and MRI can be indicative for SBA but, the diagnosis of certainty is mostly obtained incidentally during exploratory laparotomy performed for intestinal obstruction or perforation in CD patients (3). To date, the diagnostic sensitivity, specificity, and accuracy of cross-sectional imaging techniques for the detection of SBA in presence of IBD are unknown and scarcely investigated. When performed with the indication of studying the small bowel using both CT and MRI can be pursued with enteroclysis or enterography. CT usually demonstrates a small bowel obstruction, single or multiple luminal strictures and eventually perforation (44). A wall thickening with irregular abrupt margins can be observed, as well as an upstream jejunal or ileal dilatation, an annular or nodular morphology of the lesion, contrastenhancement, an involvement of the mesenteric margin, and locoregional lymphadenopathies (44). These features have been reported in a retrospective series of twenty-four CD patients affected by SBA (43). The authors assessed a perforation rate around $25 \%$ at the time of imaging and, combined together, approximately $70 \%$ of the CD-SBA patients had either a mass, a perforation, an abscess, or distant secondary locations, suggesting that these findings should rise the suspicion of a probable superimposed SBA at cross-sectional imaging evaluation of CD patients (44). A further small series in which CT-enterography was investigated as diagnostic method for SBA in CD, four different radiological patterns were described: (I) a small bowel mass, (II) a long stricture with heterogeneous submucosal signal, (III) a short and high-grade stenosis with upstream dilatation, and (IV) an asymmetric circumferential wall thickening (45). In addition to CT, the adoption of $\mathrm{PET} / \mathrm{CT}$ has been described for the diagnosis of SBA at the initial work-up $(45,46)$. This technique, despite being slightly inaccurate with respect to the localization of lesions, presents together and simultaneously functional and morphological information $(46,47)$. Regarding MRI, specifically MR enterography, it can show a circumferential and asymmetric as well as irregular bowel thickening with associated stranding of the surrounding mesenteric fat and luminal narrowing (48). Alternatively, the tumor can present 

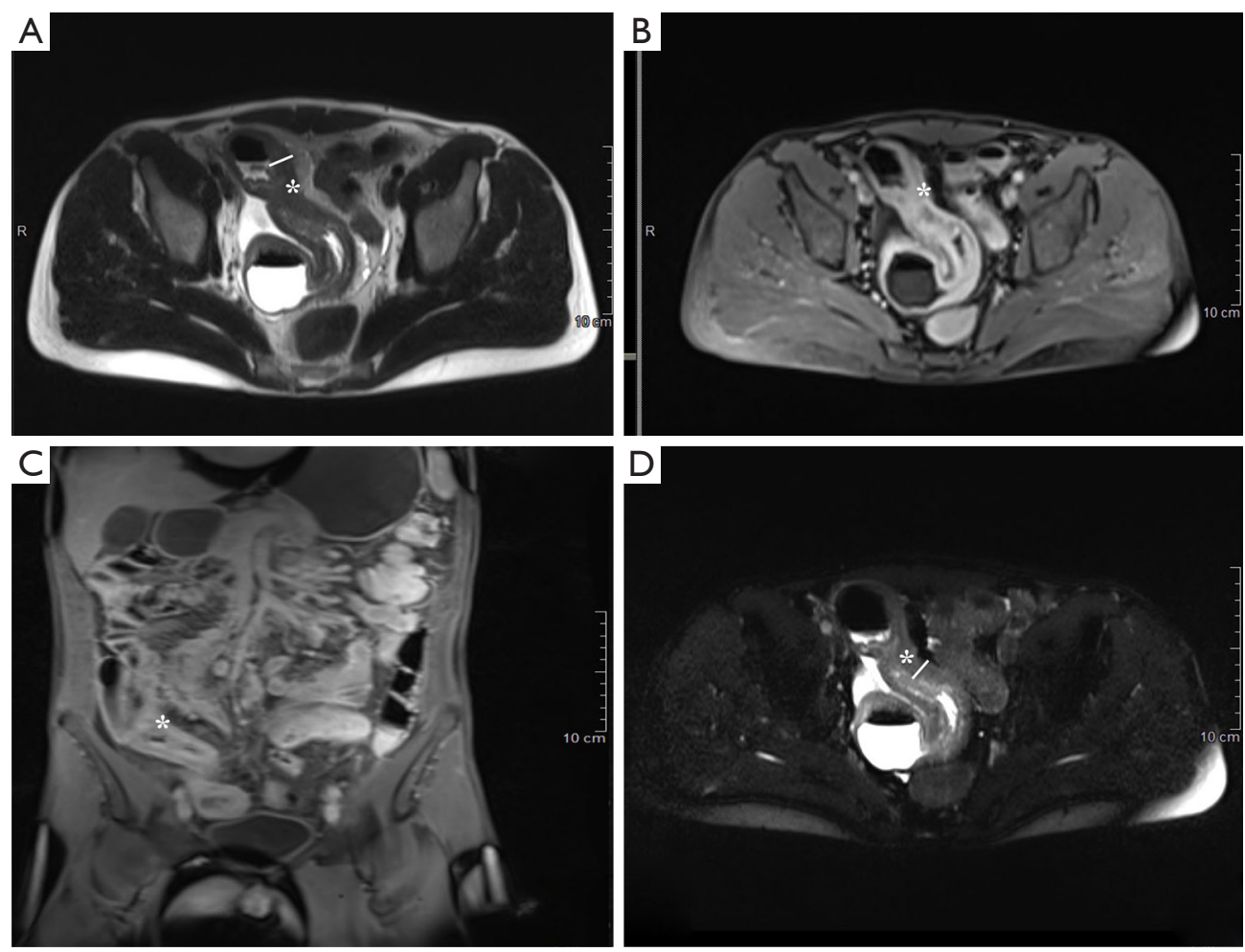

Figure 2 An adenocarcinoma of the small bowel overlapping an active Crohn's disease of the terminal ileum on magnetic resonance imaging (MRI). (A) Axial T2; (B) axial T1 with fat suppression; (C) coronal T1 with fat suppression; (D) axial T2 with fat suppression. *, identifies the neoplastic lesion; the white lines indicate the wall thickening.

as a tumor mass extending to surrounding structures (i.e., the ileocecal valve, the confining aspect of the cecum) with high $b$ coefficient (expressed as $\mathrm{s} / \mathrm{mm}^{2}$ identifies the diffusion encoding strength) values indicating a restricted diffusion at DWI (48). A case of SBA superimposed to active CD at MR enterography is shown in Figure 2. There are no data about BUS and small bowel adenocarcinoma. Traditionally the small bowel requires exceptional skills and is for its nature difficult to be explored, this may partially explain the lack of studies in the field (33).

\section{Perianal and anal cancer}

Anal squamous-cell carcinoma (ASC) occurs as unfrequently as 0.01 to 0.02 per 1,000 person-years both in patients with IBD and in the general population (49). However, in the context of a long-standing, more than 10 years, fistulizing perianal $\mathrm{CD}$, the incidence can increase by ten times and is estimated by 0.2 per 1,000 patient-years (50). Adenocarcinomas or squamous-cell carcinomas are associated with fistulizing perianal disease. They are pathogenetically unrelated to human papillomavirus (HPV) infection (50). Since the clinical presentation can be largely nonspecific and the visualization of the anal lesions can be prevented by a stenosis or a complex disease limiting an adequate examination, the diagnosis of ASC is frequently delayed (51). Dedicated gastroenterologists and surgeons are usually warned in case of an abrupt change symptoms or an unjustified pain (51). Generally, patients developing fistulaassociated cancers have an extremely poor prognosis (51).

\section{$C T$ and MRI}

MRI and CT provide a detailed imaging of the anal region allowing to detect and define the extent of neoplastic lesions, characterizing any concomitant nodal and visceral metastases, being the recommended methods by oncological societies for the diagnosis and staging of anal cancer (52). Among the diagnostic imaging techniques, CT and MRI are traditionally used for the early detection of ASC, despite having intermediate to low sensitivity varying from $58 \%$ to $75 \%(53,54)$. With respect to the radiological appearance, 

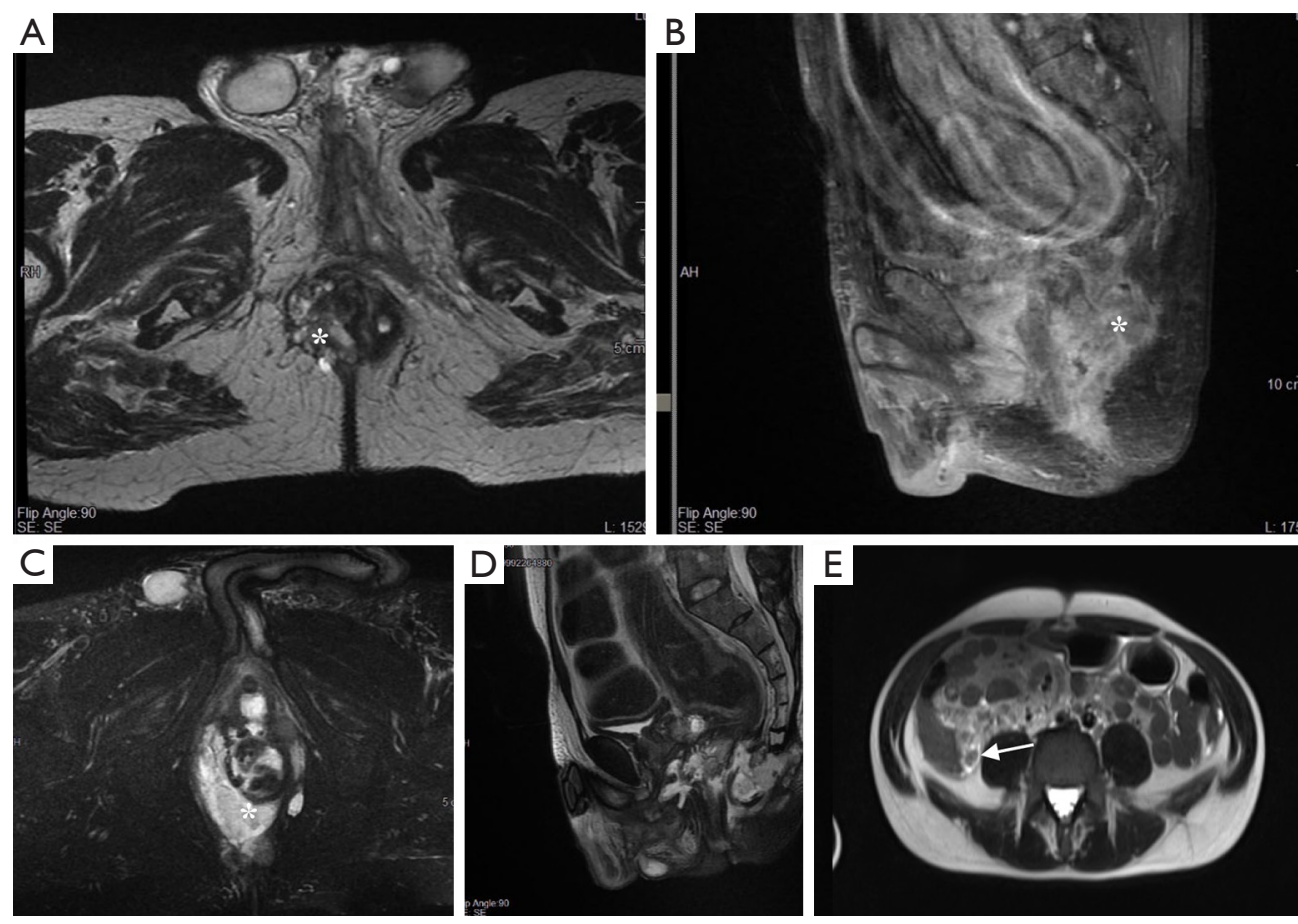

Figure 3 An anal adenocarcinoma occurring on a fistula in a patient affected by peri-anal Crohn's disease. (A) Axial T2; (B) sagittal T1 with fat suppression after gadolinium; (C) axial T2 with fat suppression; (D) sagittal T2; (E) axial T2. *, identifies the neoplastic lesion; the arrow indicates local lymph nodes.

in a small retrospective series of CD patients with perianal cancer, two main features of ASC were reported: the tumoral tissue showed a relatively less intense enhancement compared to the areas of pure inflammation and fistulas harboring malignant transformation showed a more severe thickening than chronic quiescent fistulas (55). Dynamic contrast-enhanced MRI combined with T2 sequences more accurately allowed the integration of the above-mentioned features (55). In the same study, four cases of mucinous adenocarcinoma of the anal region were investigated through MRI with the recognition of a specific pattern of lobulated fluid cavities with a rather delayed enhancement. None of the controls displayed this characteristic (55). The neoplastic tissue in the anal canal was extensively described by Taolini et al. as having a low-to-intermediate T1 signal intensity and a positive enhancement after intravenous administration of gadolinium (56). Conversely, anal neoplasms exhibited an intermediate signal intensity on T2weighted and short and medium-TI (STIR) sequences (56). As regards CT scan, ASC appeared as solid masses within the anus displaying a heterogeneous enhancement (56). Finally, 18-fluorodeoxyglucose positron emission tomography (18-FDG PET/CT) has a relevant role in the staging evaluation, as around $98 \%$ of anal cancers mirrors FDG-avidity $(57,58)$. Still, the specificity of this technique is reduced in case of severely active inflammation that might result in a false positive result. A case of fistulaassociated anal cancer in a CD patient is shown in Figure 3. If the radiological findings are either inconclusive or highly suspicious, the biopsy of the lesion should be considered and performed eventually under anesthesia (51).

\section{Endoanal and TPUS}

Endoanal ultrasound (EAUS) examination is possible with predisposed 5 to $12 \mathrm{MHz}$ endoprobes transducing radially, linearly or as curved array (59). This method enables a detailed evaluation of the anal canal and the distinction between benign and malignant diseases (59). As for conventional ultrasonographic assessment, adjunctive tools such as color Doppler, contrast enhancement and elastography can be used simultaneously (59). EUAS is essential in the locoregional staging of ASC, specifically with respect to $\mathrm{T}$ and $\mathrm{N}$ parameters, in the pre-treatment 

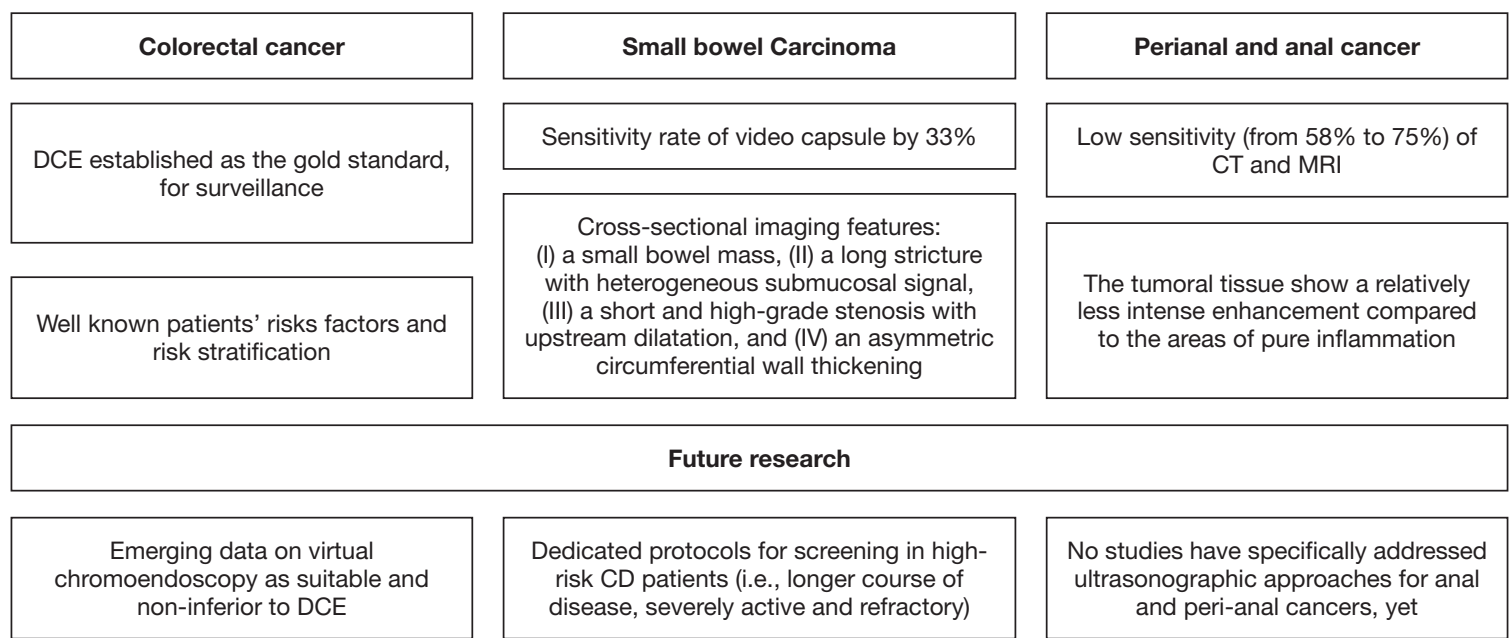

Future research

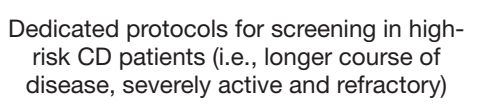

No studies have specifically addressed ultrasonographic approaches for anal and peri-anal cancers, yet

Figure 4 Clinical implications of imaging techniques and unaddressed issues in the diagnosis of carcinomas in IBD patients. IBD, inflammatory bowel diseases; DCE, dye-chromoendoscopy; CT, computed tomography; MRI, magnetic resonance imaging; CD, Crohn's disease.

assessment, after therapy and in the follow-up. It has been demonstrated that EAUS and MRI have similar accuracy in assessing the local extension $(60,61)$. Additionally, TPUS can be adopted as an easy, noninvasive, and accurate examination. Unfortunately, the technique is not very widespread yet and the data regarding its application in the field of carcinomas in IBD are unavailable. Whereas the evidence on TPUS as concerns the diagnosis and monitoring of perianal fistulas and abscesses is more robust and growing (62). To date, no studies have specifically addressed these ultrasonographic approaches in the field of ASCC superimposed to IBD.

\section{Discussion}

This review illustrates the current evidence on imaging in the field of cancer arising on IBD. Gastrointestinal and extra-intestinal cancers represent a major complication in IBD patients and a challenge for dedicated physicians (3-5). Different incidence rates exist depending on the location and duration of the disease and the subtype activity (3-5). Several imaging techniques are available and can be chosen based on their accuracy and on the site that has to be investigated. Figure 4 summarizes the clinical implications of imaging techniques and the unaddressed issues in the diagnosis of carcinomas in IBD patients. What emerges from our review is that robust studies comparing radiological appearance with histology are lacking and, overall, the sensitivity and specificity of the different techniques in distinguishing a location of IBD from an overlapping tumor are unknown. Indeed, randomized clinical trials have been conducted only with respect to endoscopy in the field of CRC surveillance, conversely, the exposed data on cross-sectional imaging derive mainly from retrospective cohorts and few prospective studies, thus limiting the quality of the data themselves. Many largely non-specific radiological features have been described both for CRC and SBA at cross-sectional imaging (i.e., freefluid effusion, irregular margins, loss of wall stratification, a mass, a perforation) $(28,29,44)$. The scientific community recognizes a sub-classification with two radiological patterns (type 1 and type 2) for IBD-related CRC $(10,28)$ that may guide the radiologists in their interpretation. As concerns BUS the loss of ultrasonographic stratification has been indicated as highly specific and statistically significant compared to active IBD $(\mathrm{P}<0.001)$ (33), however in our view, this parameter might be unreliable in case of severe disease where the echo pattern may appear as completely loss or disrupted.

As far as we are concerned, the highest accuracy in distinguishing a malignant lesion from a site of active IBD is probably obtained by the combination of many different imaging characteristics, such as the symmetry, the margins, and the contrast enhancement, that can be integrated together to endorse the suspicion of malignancy and maximize the diagnostic yield. A further issue regards surveillance, that is standardized and widespread for CRC since the evolution from dysplasia to carcinoma and the 
biology of tumorigenesis is better understood. Current recommendations indicate to proceed with DCE starting from 8 years of disease and with subsequent timing intervals depending on the risk factors (7). In particular, according to the current recommendations, IBD patients with coexisting PSC should undergo annual surveillance colonoscopy regardless of duration, extent and activity of disease (63). The intervals following surveillance are established as follows: in case of high-risk (i.e., stricture, previous dysplasia, extensive colitis) it is indicated after 1 year, in case of intermediate risk the suggested schedule is after 2 to 3 years, and finally patients without risk factors are scheduled after 5 years (63). The progression of dysplastic foci to SBA and to anal cancer in patients with $\mathrm{CD}$ is yet to be established preventing the development of dedicated surveillance programs. Protocols elucidating on the appropriate frequency and imaging modality are warranted both for SBA and ASC, in order to expedite an early diagnosis in these highly lethal cancers. Considering the scarce evidence in this field a multidisciplinary discussion and management with surgeons, radiologists and oncologists of CD and UC patients with suspected neoplastic lesions appears mandatory, eventually counseling these patients for prompt biopsy or surgical exploration.

\section{Acknowledgments}

Funding: None.

\section{Footnote}

Provenance and Peer Review: This article was commissioned by the Guest Editors (Francesco Giudici and Gabriele Dragoni) for the series "Carcinomas Development in Inflammatory Bowel Diseases" published in Chinese Clinical Oncology. The article has undergone external peer review.

Reporting Checklist: The authors have completed the Narrative Review reporting checklist. Available at https:// cco.amegroups.com/article/view/10.21037/cco-21-98/rc

Conflicts of Interest: All authors have completed the ICMJE uniform disclosure form (available at https://cco.amegroups. com/article/view/10.21037/cco-21-98/coif). The series "Carcinomas Development in Inflammatory Bowel Diseases" was commissioned by the editorial office without any funding or sponsorship. FF has received consulting fees, payment or honoraria lectures, presentations, speakers bureaus, manuscript writing or educational events, payment for expert testimony, support for attending meetings and/ or travel, patents planned, issued or pending, leadership or fiduciary role in other board, society, committee or advocacy group, paid or unpaid from Janssen. MA received royalties or licenses, consulting fees, payment or honoraria for lectures, presentations, speakers bureaus, manuscript writing or educational events, payment for expert testimony, support for attending meetings and/or travel, patents planned, issued or pending, participation on a Data Safety Monitoring Board or Advisory Board, leadership or fiduciary role in other board, society, committee or advocacy group, paid or unpaid, from Nikkiso Europe, Janssen, Abbvie and Pfizer. GF received royalties or licenses, consulting fees, payment or honoraria for lectures, presentations, speakers bureaus, manuscript writing or educational events, payment for expert testimony, participation on a Data Safety Monitoring Board or Advisory Board, leadership or fiduciary role in other board, society, committee or advocacy group, paid or unpaid, from MSD, Takeda Pharmaceuticals, AbbVie, Pfizer, Celltrion, Amgen, Sandoz, Samsung and Janssen Pharmaceuticals. SD received royalties or licenses, consulting fees, payment or honoraria for lectures, presentations, speakers bureaus, manuscript writing or educational events, payment for expert testimony, support for attending meetings and/or travel, patents planned, issued or pending, participation on a Data Safety Monitoring Board or Advisory Board, leadership or fiduciary role in other board, society, committee or advocacy group, paid or unpaid, from Schering- Plough, AbbVie, MSD, UCB Pharma, Ferring, Cellerix, Millenium Takeda, Nycomed, Pharmacosmos, Actelion, Alphawasserman, Genentech, Grunenthal, Pfizer, Astra Zeneca, Novo Nordisk, Cosmo Pharmaceuticals, Vifor and Johnson \& Johnson, Nikkiso Europe GMBH, Theravance. The authors have no other conflicts of interest to declare.

Ethical Statement: The authors are accountable for all aspects of the work in ensuring that questions related to the accuracy or integrity of any part of the work are appropriately investigated and resolved.

Open Access Statement: This is an Open Access article distributed in accordance with the Creative Commons Attribution-NonCommercial-NoDerivs 4.0 International License (CC BY-NC-ND 4.0), which permits the noncommercial replication and distribution of the article with 
the strict proviso that no changes or edits are made and the original work is properly cited (including links to both the formal publication through the relevant DOI and the license). See: https://creativecommons.org/licenses/by-nc-nd/4.0/.

\section{References}

1. Ungaro R, Mehandru S, Allen PB, et al. Ulcerative colitis. Lancet 2017;389:1756-70.

2. Torres J, Mehandru S, Colombel JF, et al. Crohn's disease. Lancet 2017;389:1741-55.

3. Beaugerie L, Itzkowitz SH. Cancers complicating inflammatory bowel disease. $\mathrm{N}$ Engl J Med 2015;372:1441-52.

4. Magro F, Peyrin-Biroulet L, Sokol H, et al. Extra-intestinal malignancies in inflammatory bowel disease: results of the 3rd ECCO Pathogenesis Scientific Workshop (III). J Crohns Colitis 2014;8:31-44.

5. Kappelman MD, Farkas DK, Long MD, et al. Risk of cancer in patients with inflammatory bowel diseases: a nationwide population-based cohort study with 30 years of follow-up evaluation. Clin Gastroenterol Hepatol 2014;12:265-73.e1.

6. Scharl S, Barthel C, Rossel JB, et al. Malignancies in Inflammatory Bowel Disease: Frequency, Incidence and Risk Factors-Results from the Swiss IBD Cohort Study. Am J Gastroenterol 2019;114:116-26.

7. Laine L, Kaltenbach T, Barkun A, et al. SCENIC international consensus statement on surveillance and management of dysplasia in inflammatory bowel disease. Gastroenterology 2015;148:639-651.e28.

8. Jess T, Simonsen J, Jørgensen KT, et al. Decreasing risk of colorectal cancer in patients with inflammatory bowel disease over 30 years. Gastroenterology 2012;143:375-81. e1; quiz e13-4.

9. Bettenworth D, Bokemeyer A, Baker M, et al. Assessment of Crohn's disease-associated small bowel strictures and fibrosis on cross-sectional imaging: a systematic review. Gut 2019;68:1115-26.

10. Barral M, Hoeffel C, Boudiaf M, et al. Rectal cancer in inflammatory bowel diseases: MR imaging findings. Abdom Imaging 2014;39:443-51.

11. Lopez A, Pouillon L, Beaugerie L, et al. Colorectal cancer prevention in patients with ulcerative colitis. Best Pract Res Clin Gastroenterol 2018;32-33:103-9.

12. Jess T, Rungoe C, Peyrin-Biroulet L. Risk of colorectal cancer in patients with ulcerative colitis: a meta-analysis of population-based cohort studies. Clin Gastroenterol
Hepatol 2012;10:639-45.

13. Rutter MD, Saunders BP, Wilkinson KH, et al. Thirtyyear analysis of a colonoscopic surveillance program for neoplasia in ulcerative colitis. Gastroenterology 2006;130:1030-8.

14. Itzkowitz SH, Yio X. Inflammation and cancer IV. Colorectal cancer in inflammatory bowel disease: the role of inflammation. Am J Physiol Gastrointest Liver Physiol 2004;287:G7-17.

15. Bye WA, Ma C, Nguyen TM, et al. Strategies for Detecting Colorectal Cancer in Patients with Inflammatory Bowel Disease: A Cochrane Systematic Review and MetaAnalysis. Am J Gastroenterol 2018;113:1801-9.

16. Iacucci $M$, Uraoka T, Fort Gasia $M$, et al. Novel diagnostic and therapeutic techniques for surveillance of dysplasia in patients with inflammatory bowel disease. Can J

Gastroenterol Hepatol 2014;28:361-70.

17. Marion JF, Waye JD, Present DH, et al. Chromoendoscopy-targeted biopsies are superior to standard colonoscopic surveillance for detecting dysplasia in inflammatory bowel disease patients: a prospective endoscopic trial. Am J Gastroenterol 2008;103:2342-9.

18. Moussata D, Allez M, Cazals-Hatem D, et al. Are random biopsies still useful for the detection of neoplasia in patients with IBD undergoing surveillance colonoscopy with chromoendoscopy? Gut 2018;67:616-24.

19. van den Broek FJ, Fockens P, van Eeden S, et al. Endoscopic tri-modal imaging for surveillance in ulcerative colitis: randomised comparison of high-resolution endoscopy and autofluorescence imaging for neoplasia detection; and evaluation of narrow-band imaging for classification of lesions. Gut 2008;57:1083-9.

20. Iacucci M, Kaplan GG, Panaccione R, et al. A Randomized Trial Comparing High Definition Colonoscopy Alone With High Definition Dye Spraying and Electronic Virtual Chromoendoscopy for Detection of Colonic Neoplastic Lesions During IBD Surveillance Colonoscopy. Am J Gastroenterol 2018;113:225-34.

21. El-Dallal M, Chen Y, Lin Q, et al. Meta-analysis of Virtual-based Chromoendoscopy Compared With Dyespraying Chromoendoscopy Standard and High-definition White Light Endoscopy in Patients With Inflammatory Bowel Disease at Increased Risk of Colon Cancer. Inflamm Bowel Dis 2020;26:1319-29.

22. Endoscopic Classification Review Group. Update on the paris classification of superficial neoplastic lesions in the digestive tract. Endoscopy 2005;37:570-8.

23. Kudo S, Tamura S, Nakajima T, et al. Diagnosis of 
colorectal tumorous lesions by magnifying endoscopy. Gastrointest Endosc 1996;44:8-14.

24. Benson AB, Venook AP, Al-Hawary MM, et al. NCCN Guidelines Insights: Colon Cancer, Version 2.2018. J Natl Compr Canc Netw 2018;16:359-69.

25. Benson AB, Venook AP, Al-Hawary MM, et al. Rectal Cancer, Version 2.2018, NCCN Clinical Practice Guidelines in Oncology. J Natl Compr Canc Netw 2018;16:874-901.

26. Ganguly A, Meredith S, Probert C, et al. Colorectal cancer mimics: a review of the usual suspects with pathology correlation. Abdom Radiol (NY) 2016;41:1851-66.

27. Wang X, Yuan M, Mi H, et al. The feasibility of differentiating colorectal cancer from normal and inflammatory thickening colon wall using CT texture analysis. Sci Rep 2020;10:6346.

28. Hristova L, Soyer P, Hoeffel C, et al. Colorectal cancer in inflammatory bowel diseases: CT features with pathological correlation. Abdom Imaging 2013;38:421-35.

29. Regge D, Neri E, Turini F, et al. Role of CT colonography in inflammatory bowel disease. Eur J Radiol 2009;69:404-8.

30. Bonaventure T, Goéré D, Boige V, et al. Beware of PETCT results in cases of cancer occurring in the setting of chronic inflammatory bowel disease. J Chir (Paris) 2009;146:579-82.

31. Yu H, Feng C, Wang Z, et al. Potential of diffusionweighted imaging in magnetic resonance enterography to identify neoplasms in the ileocecal region: Use of ultrahigh b-value diffusion-weighted imaging. Oncol Lett 2019;18:1451-7.

32. Avcu S, Bulut MD, Yavuz A, et al. Value of DW-MRI ADC quantification of colonic wall lesions in differentiation of inflammatory bowel disease and colorectal carcinoma. Jpn J Radiol 2014;32:6-13.

33. Maconi G, Nylund K, Ripolles T, et al. EFSUMB Recommendations and Clinical Guidelines for Intestinal Ultrasound (GIUS) in Inflammatory Bowel Diseases. Ultraschall Med 2018;39:304-17.

34. Zhang F, Miao LY, Ge HY, et al. Usefulness of ContrastEnhanced Ultrasound in Differentiating Inflammatory Bowel Disease From Colon Cancer. Ultrasound Med Biol 2018;44:124-33.

35. Jess T, Gamborg M, Matzen P, et al. Increased risk of intestinal cancer in Crohn's disease: a meta-analysis of population-based cohort studies. Am J Gastroenterol 2005;100:2724-9.

36. Elriz K, Carrat F, Carbonnel F, et al. Incidence, presentation, and prognosis of small bowel adenocarcinoma in patients with small bowel Crohn's disease: a prospective observational study. Inflamm Bowel Dis 2013;19:1823-6.

37. Huang Z, Liu X, Yang F, et al. Diagnostic efficacy of double-balloon enteroscopy in patients with suspected isolated small bowel Crohn's disease. BMC Gastroenterol 2020;20:42.

38. Kodaira C, Osawa S, Mochizuki C, et al. A case of small bowel adenocarcinoma in a patient with Crohn's disease detected by PET/CT and double-balloon enteroscopy. World J Gastroenterol 2009;15:1774-8.

39. Stier MW, Paramsothy S, Dalal S. Ten-Year Retained Video Capsule With Crohn's-Associated SmallBowel Adenocarcinoma. Clin Gastroenterol Hepatol 2017;15:A29-30.

40. Cave D, Legnani P, de Franchis R, et al. ICCE consensus for capsule retention. Endoscopy 2005;37:1065-7.

41. Rondonotti E, Spada C, Adler S, et al. Small-bowel capsule endoscopy and device-assisted enteroscopy for diagnosis and treatment of small-bowel disorders: European Society of Gastrointestinal Endoscopy (ESGE) Technical Review. Endoscopy 2018;50:423-46.

42. Palascak-Juif V, Bouvier AM, Cosnes J, et al. Small bowel adenocarcinoma in patients with Crohn's disease compared with small bowel adenocarcinoma de novo. Inflamm Bowel Dis 2005;11:828-32.

43. Simon M, Cosnes J, Gornet JM, et al. Endoscopic Detection of Small Bowel Dysplasia and Adenocarcinoma in Crohn's Disease: A Prospective Cohort-Study in HighRisk Patients. J Crohns Colitis 2017;11:47-52.

44. Weber NK, Fletcher JG, Fidler JL, et al. Clinical characteristics and imaging features of small bowel adenocarcinomas in Crohn's disease. Abdom Imaging 2015;40:1060-7.

45. Soyer P, Hristova L, Boudghène F, et al. Small bowel adenocarcinoma in Crohn disease: CT-enterography features with pathological correlation. Abdom Imaging 2012;37:338-49.

46. Yoshikawa T, Takehara Y, Kikuyama M, et al. Computed tomographic enteroclysis with air and virtual enteroscopy: protocol and feasibility for small bowel evaluation. Dig Liver Dis 2012;44:297-302.

47. Paparo F, Piccardo A, Clavarezza M, et al. Computed tomography enterography and 18F-FDG PET/CT features of primary signet ring cell carcinoma of the small bowel in a patient with Crohn's disease. Clin Imaging 2013;37:794-7.

48. Placé V, Hristova L, Dray X, et al. Ileal adenocarcinoma 
in Crohn's disease: magnetic resonance enterography features. Clin Imaging 2012;36:24-8.

49. Slesser AA, Bhangu A, Bower M, et al. A systematic review of anal squamous cell carcinoma in inflammatory bowel disease. Surg Oncol 2013;22:230-7.

50. Laukoetter MG, Mennigen R, Hannig CM, et al. Intestinal cancer risk in Crohn's disease: a meta-analysis. J Gastrointest Surg 2011;15:576-83.

51. Kotze PG, Shen B, Lightner A, et al. Modern management of perianal fistulas in Crohn's disease: future directions. Gut 2018;67:1181-94.

52. Glynne-Jones R, Northover JM, Cervantes A, et al. Anal cancer: ESMO Clinical Practice Guidelines for diagnosis, treatment and follow-up. Ann Oncol 2010;21 Suppl 5:v87-92.

53. Devon KM, Brown CJ, Burnstein M, et al. Cancer of the anus complicating perianal Crohn's disease. Dis Colon Rectum 2009;52:211-6.

54. Iesalnieks I, Gaertner WB, Glass H, et al. Fistulaassociated anal adenocarcinoma in Crohn's disease. Inflamm Bowel Dis 2010;16:1643-8.

55. Lad SV, Haider MA, Brown CJ, et al. MRI appearance of perianal carcinoma in Crohn's disease. J Magn Reson Imaging 2007;26:1659-62.

56. Tonolini M, Bianco R. MRI and CT of anal carcinoma: a pictorial review. Insights Imaging 2013;4:53-62.

57. Saboo SS, Zukotynski K, Shinagare AB, et al. Anal

Cite this article as: Furfaro F, Dal Buono A, Sicuso C, Allocca M, D’Amico F, Zilli A, Fiorino G, Gabbiadini R, Danese S. Carcinomas in inflammatory bowel disease: a narrative review on diagnostic imaging techniques. Chin Clin Oncol 2022;11(3):22. doi: 10.21037/cco-21-98 carcinoma: FDG PET/CT in staging, response evaluation, and follow-up. Abdom Imaging 2013;38:728-35.

58. Agarwal A, Marcus C, Xiao J, et al. FDG PET/CT in the management of colorectal and anal cancers. AJR Am J Roentgenol 2014;203:1109-19.

59. Nuernberg D, Saftoiu A, Barreiros AP, et al. EFSUMB Recommendations for Gastrointestinal Ultrasound Part 3: Endorectal, Endoanal and Perineal Ultrasound. Ultrasound Int Open 2019;5:E34-51.

60. Otto SD, Lee L, Buhr HJ, et al. Staging anal cancer: prospective comparison of transanal endoscopic ultrasound and magnetic resonance imaging. J Gastrointest Surg 2009;13:1292-8.

61. Parikh J, Shaw A, Grant LA, et al. Anal carcinomas: the role of endoanal ultrasound and magnetic resonance imaging in staging, response evaluation and follow-up. Eur Radiol 2011;21:776-85.

62. Sagami S, Kobayashi T, Aihara K, et al. Transperineal ultrasound predicts endoscopic and histological healing in ulcerative colitis. Aliment Pharmacol Ther 2020;51:1373-83.

63. Magro F, Gionchetti P, Eliakim R, et al. Third European Evidence-based Consensus on Diagnosis and Management of Ulcerative Colitis. Part 1: Definitions, Diagnosis, Extraintestinal Manifestations, Pregnancy, Cancer Surveillance, Surgery, and Ileo-anal Pouch Disorders. J Crohns Colitis 2017;11:649-70. 